\title{
MODELADO Y SIMULACIÓN DEL SAR DEBIDO A LOS CAMPOS ELECTROMAGNÉTICOS RADIADOS POR TELÉFONOS CELULARES
}

\author{
Mario Zamorano L. ${ }^{1} \quad$ Héctor Torres S. $^{1}$ Máximo Soto E. ${ }^{1}$ \\ Recibido el 9 de marzo de 2005, aceptado el 25 de julio de 2005
}

\begin{abstract}
RESUMEN
Se presenta un modelo bioplasmático quiral que caracteriza la cabeza humana y se usa para analizar su comportamiento cuando es radiada por las microondas de la telefonía celular. La técnica FDTD permite calcular numéricamente los campos electromagnéticos y simular el coeficiente SAR. Los resultados obtenidos muestran el SAR en función de la potencia de entrada, de la impedancia de antena y de la quiralidad. La conclusión más importante es que, considerando el factor quiral del medio biológico, existe mayor absorción de la radiación emitida por los teléfonos celulares con respecto a los resultados de los modelos clásicos.
\end{abstract}

Palabras clave: Telefonía celular, quiralidad, FDTD, SAR.

\section{ABSTRACT}

A model formed by chiral bioplasma, which represents the human head inner structure and used to analyze its behavior when it is radiated by a microwave from cellular phones is presented. The FDTD technique is used to calculate the electromagnetic fields, and to simulate the SAR. The results show the SAR behavior in function of input power, antenna impedance and the chirality factor. More important conclusion is that, in considering the chiral factor of biological media, the absorption of the electromagnetic fields from cellular phones is higher than classical model.

Keywords: Cellular phones, chirality, FDTD, SAR.

\section{INTRODUCCIÓN}

En la última década se ha producido un crecimiento explosivo de la comunicación inalámbrica, particularmente el uso de telefonía celular como un enlace de gran envergadura. El sistema móvil global de comunicaciones, GSM, ha sido identificado como una posible fuente dañina para la salud humana. Se sabe que los campos electromagnéticos (CEM) de la telefonía celular, 900 y $1800 \mathrm{MHz}$, penetran los tejidos expuestos y absorben la radiación de microondas (MW). La cabeza de los usuarios está sometida a una exposición altamente localizada de MW [1]. Aunque existe gran cantidad de trabajos en el área, aún no hay un conocimiento suficientemente completo sobre este tema en particular. Sin embargo, hay un consenso general [2], [3] acerca de la importancia de evaluar correctamente los mecanismos de interacción entre los campos electromagnéticos y los sistemas biológicos.
A base de los trabajos previos desarrollados por los autores [4]-[9], se presenta un modelo bioplasmático quiral que modela, simula y evalúa la absorción, por parte de la cabeza humana, de las MW emitidas por los teléfonos celulares. En la siguiente sección se presentan las características del modelo propuesto, a continuación se determinan los CEM al interior de la cabeza y finalmente se presenta la simulación del parámetro SAR y la discusión de los resultados obtenidos.

\section{MODELADO BIOELECTROMAGNÉTICO}

Los estudios, desde el punto de vista bioelectromagnético, se tornan complejos por el hecho de que los teléfonos móviles son digitales, pulsados y modulados a baja frecuencia, cuyas formas de onda dan como resultado que corrientes iónicas y campos electromagnéticos producidos en la zona irradiada del cerebro, interactúan con la microonda del celular y el tejido cerebral, de manera no

1 Departamento de Electrónica, Universidad de Tarapacá. Casilla 6-D, 18 de Septiembre 2222, Arica, Chile, mhzlucero@uta.cl ; htorres@uta.cl 
conocida exactamente. Por lo tanto, es esencial saber qué características o conjunto de parámetros de la radiación son la causa fundamental de los efectos mencionados.

La figura 1 muestra las 6 etapas del proceso que permiten modelar, simular y evaluar la absorción de la radiación de microondas de los sistemas celulares.

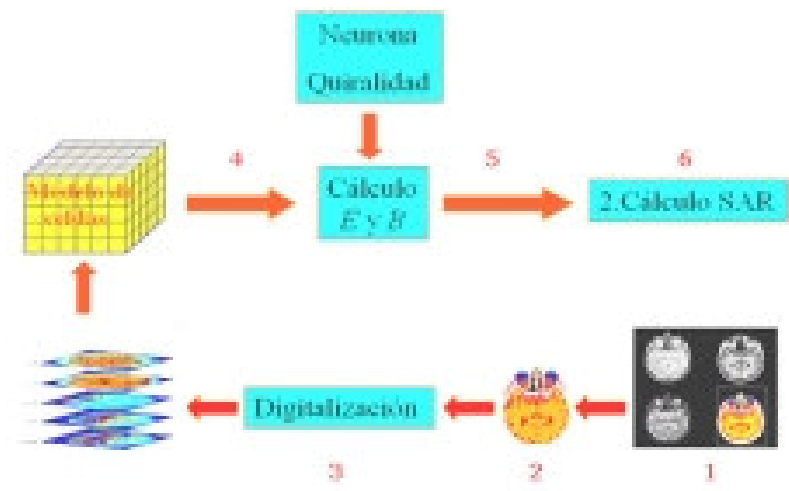

Fig. 1 Diagrama en bloque del proceso para el cálculo del coeficiente SAR.

El conocimiento detallado de la estructura interna de la cabeza ha permitido normalizar los tejidos que la componen y determinar sus propiedades eléctricas (conductividad y permitividad relativas) y densidad de masa. Importante ha sido el uso de la resonancia magnética para modelar y conocer los detalles internos de la cabeza y presentar sus características mediante imágenes individuales, imágenes MRI (Magnetic Resonance Image). La cabeza humana, según la resolución deseada, puede ser modelada por un conjunto de hasta 108 imágenes. Ver [10]-[11] para detalles de imágenes MRI. Dado que los tejidos biológicos son estructuras heterogéneas complejas, es conveniente realizar su modelado y simulación mediante la técnica FDTD por las ventajas que ella ofrece. A partir de una lámina MRI, se generan los datos necesarios para llevar a cabo el modelado en detalle de cada tejido al interior de la cabeza. Es así como el modelo de cabeza humana se genera a partir de scaners (figuras) de MRI, que son transformadas en malla de diferencias finitas en el dominio del tiempo (FDTD grid). Inicialmente las imágenes gif (MRI) son convertidas a postscript para obtener los datos de los pixeles en formato ASCII. Estos datos son convertidos a enteros. Los 256 niveles en cada píxel son interpretados como uno de los 5 diferentes medios considerados: aire, piel, hueso, sangre y tejido cerebral. La cuantización de cada celda de material permite el paso de las imágenes MRI originales a imágenes discretizadas (pasos 1-3 de la Fig. 1). El programa específico que resulta de esta conversión se inserta en el programa FDTD del cálculo del SAR (pasos 4-6 de la Fig. 1).

Este modelo está constituido por 64.000 celdas cúbicas, donde cada corte o capa es de $0,5 \mathrm{~cm}$. El número total de capas que pueden ser usadas en este modelo es de 50 . Aunque el objetivo final de nuestro trabajo es usar un modelo tridimensional que considere la totalidad de las capas, hemos analizado, inicialmente, la capa 35 , por tener mayor cantidad de tejido cerebral y donde el parámetro quiral es relevante, luego se analizaron las capas $11,12,13$ y 14 , ya que la capa 13 presenta un fenómeno interesante denominado punto caliente (hot point).

Nuestra propuesta considera agregar al modelo clásico de celdas la estructura neuronal del tejido cerebral (ver Fig. 1). El citoesqueleto de cada neurona está formado, entre otros, por microtúbulos, los cuales son cilindros huecos proteicos. En su estructura se hallan dos subunidades de proteínas denominadas tubulinas (dímeros) $\alpha$ y $\beta$, ensambladas helicoidalmente, las cuales pueden tener dos configuraciones geométricas diferentes (mayores detalles en Apéndice de [4] ). Nuestra hipótesis plantea que estas configuraciones corresponden a la quiralidad hacia la izquierda y hacia la derecha respectivamente, ya que las dos conformaciones corresponden a los estados de polarización de los dímeros eléctricos. Cálculos preliminares demuestran que los valores propios de la frecuencia de estas proteínas están entre 1 y $10 \mathrm{GHz}$ (radiación de microondas), lo que corresponde a oscilación coherente dentro del microtúbulo. Macroscópicamente, como el tejido cerebral está compuesto por cúmulos de macromoléculas de proteínas, asociamos al tejido un factor electromagnético $\zeta$ (ver ecuación 1.a), que toma en cuenta el hecho de que la onda electromagnética incidente en el tejido induce una componente quiral debido a la estructura helicoidal de la macromolécula.

El estudio de los efectos térmicos y no térmicos que causa la radiación celular sobre la cabeza humana requiere analizar en detalle la absorción fundamental, de manera de obtener una indicación básica del coeficiente de absorción específica, SAR. Por ello, después de definir el modelo de cabeza a utilizar, se debe analizar en detalle la absorción fundamental producida por la radiación celular sobre que los tejidos internos que componen la cabeza humana, para lo cual se requiere evaluar los CEM incidentes.

\section{Evaluación de los CEM}

Los medios quirales son dieléctricos que se caracterizan por la asimetría de sus moléculas. Como fenómeno 
electromagnético, la quiralidad puede ser considerada como actividad óptica y corresponde a la rotación del plano de polarización. En un medio lineal isótropo dicha rotación puede predecirse por medio de las ecuaciones de Maxwell agregando, a la polarización $P$, un término proporcional a $\nabla \times E$. Las ecuaciones de Born-Fedorov permiten caracterizar el medio quiral por medio de las siguientes ecuaciones constitutivas:

$$
\begin{aligned}
& \boldsymbol{D}=\varepsilon(\boldsymbol{E}+\zeta \nabla \times \boldsymbol{E}) \\
& \boldsymbol{B}=\mu(\boldsymbol{H}+\zeta \nabla \times \boldsymbol{H})
\end{aligned}
$$

donde $\varepsilon, \mu$ y $\zeta$ son la permitividad, la permeabilidad y el pseudoescalar quiral respectivamente. Este último representa la medida de la quiralidad y tiene unidades de longitud. Se observa que el rotor del plano de la polarización se puede predecir a partir de las ecuaciones de Maxwell, considerando que el vector $P(M)$ tiene un término adicional proporcional a $\nabla \times E \nabla \times H)$ [12], [6]. Si se asume que el medio es isótropo, impermeable y no dispersivo, las componentes cartesianas del campo son:

$$
\begin{gathered}
D_{x, y, z}=\varepsilon_{0} \varepsilon_{r} E_{x, y, z}+\varepsilon_{0} \varepsilon_{r} \zeta\left[\frac{\partial E_{z, x, y}}{\partial y, z, x}-\frac{\partial E_{y, z, x}}{\partial z, x, y}\right] \\
B_{x, y, z}=\mu_{0} H_{x, y, z}+\mu_{0} \zeta\left[\frac{\partial H_{z, x, y}}{\partial y, z, x}-\frac{\partial H_{y, z, x}}{\partial z, x, y}\right]
\end{gathered}
$$

Usando el sistema de unidades MKS, las ecuaciones escalares, que se presentan a continuación son equivalentes a las ecuaciones de Maxwell en el sistema de coordenadas rectangulares $(x, y, z)$ :

$$
\begin{gathered}
\frac{\partial H_{x, y}}{\partial t}=\mp \frac{1}{\mu} \frac{\partial E_{z}}{\partial y, x} \pm \zeta \omega k_{y, x} H_{z} \\
\frac{\partial H_{z}}{\partial t}=\frac{1}{\mu}\left(\frac{\partial E_{x}}{\partial y}-\frac{\partial E_{y}}{\partial x}\right)+\zeta \omega\left(k_{x} H_{y}-k_{y} H_{x}\right) \\
\frac{\partial E_{x, y}}{\partial t}= \pm \frac{1}{\varepsilon} \frac{\partial H_{z}}{\partial y, x} \pm \zeta \omega k_{y, x} E_{z}-\sigma E_{x, y} \\
\frac{\partial E_{z}}{\partial t}=\frac{1}{\varepsilon}\left(\frac{\partial H_{y}}{\partial x}-\frac{\partial H_{x}}{\partial y}\right)+\zeta \omega\left(k_{x} E_{y}-k_{y} E_{x}\right)-\sigma E_{z}
\end{gathered}
$$

donde $\omega=2 \pi f, k_{x}=2 \pi / \lambda_{x}$ y $k_{y}=2 \pi / \lambda_{y}$.
En el sistema de ecuaciones diferenciales presentado, se observa que las componentes vectoriales del flujo eléctrico están relacionadas con las respectivas componentes del campo eléctrico y, además, son proporcionales a las derivadas parciales de sus componentes perpendiculares. La dificultad evidente de un tratamiento analítico de estas ecuaciones radica en que poseen derivadas parciales espacio-temporales, donde interviene quiralidad del medio, lo que impide que puedan ser reducidas a una ecuación diferencial tradicional cuya solución sea conocida, y es necesario, por lo tanto, recurrir a un tratamiento numérico.

\section{Discretización por FDTD y cálculo del SAR}

Teniendo presente las aproximaciones de las derivadas parciales hechas por Mur [12], para el caso de una interfase quiral-aquiral, es posible discretizar las ecuaciones anteriores mediante el algoritmo de Yee, a través del método FDTD [12]:

$$
\left.\frac{\partial^{2} U}{\partial x \partial t}\right|_{i} ^{n}=\frac{1}{2 \Delta t}\left(\left.\frac{\partial U}{\partial x}\right|_{i} ^{n+1}-\left.\frac{\partial U}{\partial x}\right|_{i} ^{n-1}\right)=\frac{1}{2 \Delta t}\left[\left(\frac{\left.U\right|_{i+1 / 2} ^{n+1}-\left.U\right|_{i-1 / 2} ^{n+1}}{\Delta x}\right)-\left(\frac{\left.U\right|_{i+1 / 2} ^{n-1}-\left.U\right|_{i-1 / 2} ^{n-1}}{\Delta x}\right)\right]
$$

Su aplicación a las ecuaciones (2)-(6) nos permite determinar los CEM que se requieren para calcular el coeficiente SAR, el que se determina de acuerdo con la siguiente ecuación [2]-[3]:

$$
S A R_{i, j}=\frac{\left.\sigma_{i, j} E_{T}^{2}\right|_{i, j}}{2 \rho_{i, j}}
$$

donde:

$$
\left.E_{T}\right|_{i, j}=\sqrt{\left.\frac{1}{n} \sum_{1}^{n} E_{y}^{2}\right|_{i, j} ^{n}+\left.E_{x}^{2}\right|_{i, j} ^{n}+\left.E_{z}^{2}\right|_{i, j} ^{n}}
$$

A fin de analizar el efecto de las ondas electromagnéticas de los teléfonos celulares, sobre la estructura interna de la cabeza, se realizaron simulaciones volumétricas considerando $\mathrm{z}=0,5 \mathrm{~cm}$. Los resultados se presentan como en estructura planar (x-y), pero son válidos hasta una profundidad de $\mathrm{z}=0,5 \mathrm{~cm}$, por lo que concuerdan con la norma ANSI/IEEE C95.1-1992. Los parámetros considerados fueron la densidad de la potencia incidente y la tasa a la cual se absorbe la energía electromagnética (SAR). La fuente de radiación del teléfono celular fue modelada por una antena dipolo equivalente $\mathrm{R}_{\mathrm{a}}$, que varía entre 40 y $180 \mathrm{ohms}$. La potencia asume los valores máximos del sistema GSM, a saber 0.25, 2 y $3.56 \mathrm{~W}$. 
Para la simulación del SAR usamos la ecuación (8). Los programas de simulación se desarrollaron para las frecuencias de 900 y $1.800 \mathrm{MHz}$; se supuso que la antena del teléfono celular produce una señal isotrópica en forma de onda plana que se propaga desde el centro de la antena y, finalmente, se consideraron 4 tipos de tejidos: piel, hueso, sangre y tejido cerebral.

\section{SIMULACIÓN Y ANÁLISIS DE RESULTADOS}

En esta sección se entregan los resultados de las simulaciones hechas para la capa 35 y para las capas de la 11 a la 14. La Fig. 2a muestra la versión digitalizada de la capa 35 , en una matriz de $100 \times 100$, de su correspondiente imagen MRI. El análisis de esta figura muestra que la capa 35 está formada, mayoritariamente, por tejido cerebral. En la Fig. 3 se presenta la variación del coeficiente SAR para un valor nulo del factor quiral, o sea, $\zeta=0$. Los resultados de esta simulación (aquiral) constituyen la base que servirá como referencia para los resultados que se obtengan cuando el cerebro sea considerado quiral, o sea, para $\zeta \neq 0$.

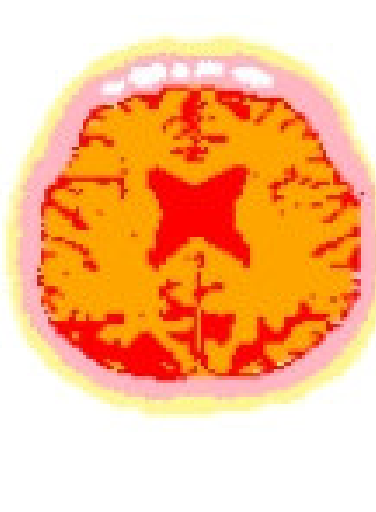

$\mathrm{a}$

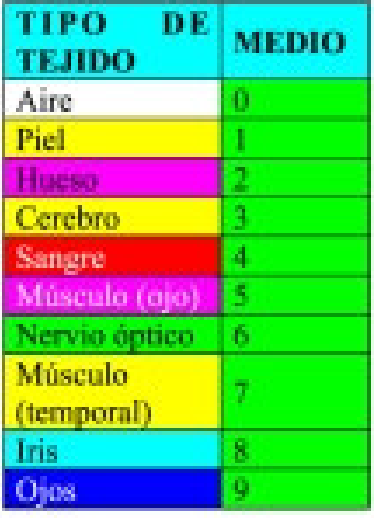

b
Fig. 2 a) Imagen digitalizada capa 35. Matriz de $100 \times 100$.

b) Tabla con relación color-tipo de tejido.

La Fig. 3 nos indica que el SAR se atenúa, rápidamente, al interior de la cabeza y la Fig. 4 demuestra que el SAR disminuye si se separa la antena de la cabeza. Si la potencia es de $0,25 \mathrm{~W}$ y la distancia cabeza-fuente aumenta de 1 a $2 \mathrm{~cm}$ se produce una disminución del $40 \%$ del SAR. Para el caso de 2 y 3,65 W la disminución es aproximadamente del $58 \%$.

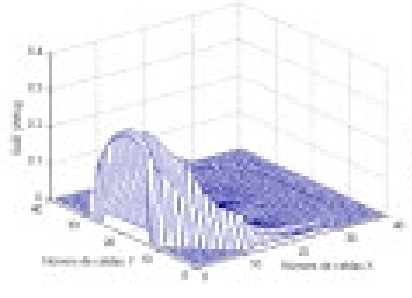

a

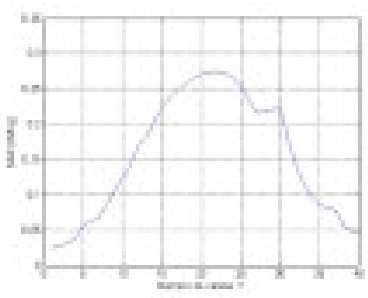

c

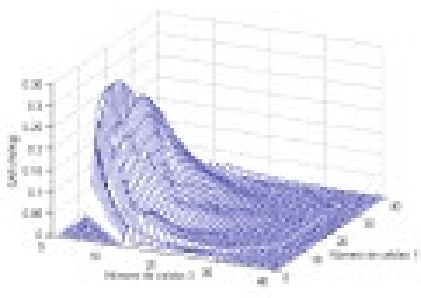

b

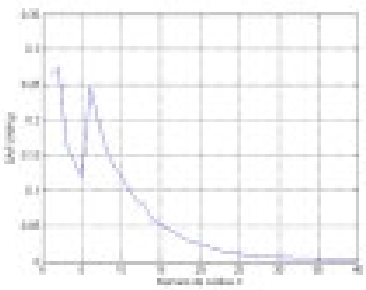

d
Fig. 3 Distribución del SAR en la capa 35 para un factor $\zeta=0$. a) en el plano XY; b) en el plano XY rotado en $90^{\circ}$; c) perfil para apreciar el valor máximo del SAR; d) perfil para ver en que celda se ubica el máximo de absorción.

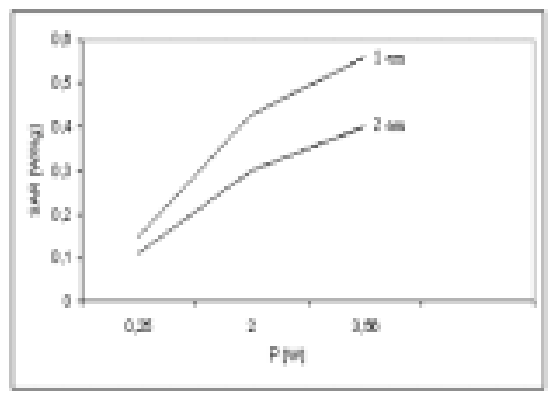

Fig. 4 SAR vs. potencia, con distancia como parámetro.

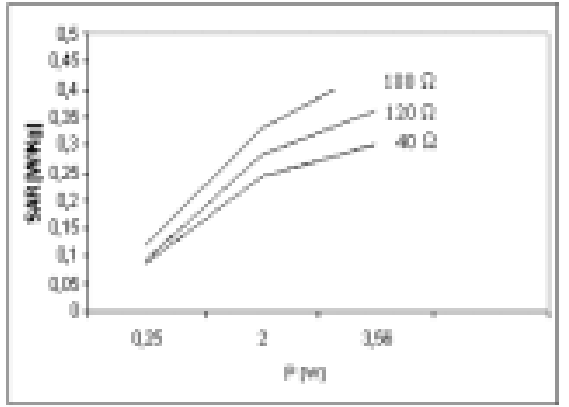

Fig. 5 SAR vs. potencia, con resistencia como parámetro. 
El aumento de la resistencia de antena significa un aumento del SAR, lo que se manifiesta en la Fig. 5. Esto se debe a que, para una potencia de operación constante, al aumentar $\mathrm{R}_{\mathrm{a}}$ aumenta también el voltaje $\mathrm{y}$, por ende, el SAR. Los valores usados fueron 40, 120 y $180 \mathrm{ohms}$. El valor máximo del SAR fue de $0,27 \mathrm{~W} / \mathrm{Kg}$ y se produce a nivel de la piel (celda $\mathrm{N}^{\circ} 1$ ). Se destaca también que el SAR, a nivel del hueso, tiene una amplitud de aproximadamente el $90 \%$ de la correspondiente a la parte externa (piel). Estos resultados, obtenidos para $\zeta=0$, son similares (aunque menor en magnitud) a los obtenidos por otros autores, [13]-[15], cuyos modelos no consideran la quiralidad del medio.

Realizando simulaciones sistemáticas, para una potencia de $3,65 \mathrm{~W}$ y una impedancia de antena de $180 \mathrm{ohms}$, se determina el perfil del SAR en función de la distancia hacia el interior de la cabeza, para variaciones entre \pm 1 del coeficiente quiral. Los resultados se muestran en la Fig. 6, donde queda de manifiesto que la absorción de las ondas de microondas emitidas por la telefonía celular aumenta cuando se considera la quiralidad del cerebro. Resultados similares se obtienen para potencias de 2 y $0,6 \mathrm{~W}$, antenas de 120 y $40 \mathrm{ohms}$. El mismo perfil de curva se obtiene para elevados valores del factor quiral, hasta $\zeta= \pm 4$. Con estas simulaciones es posible obtener el gráfico que se observa en la Fig. 7, donde se observa como la variación del factor quiral modifica el valor máximo de coeficiente SAR. Analizando esta figura se deduce que el SAR aumenta un $68 \%$ para valores de $\zeta$ pequeños y un aumento de casi tres veces para $\zeta= \pm 2$.

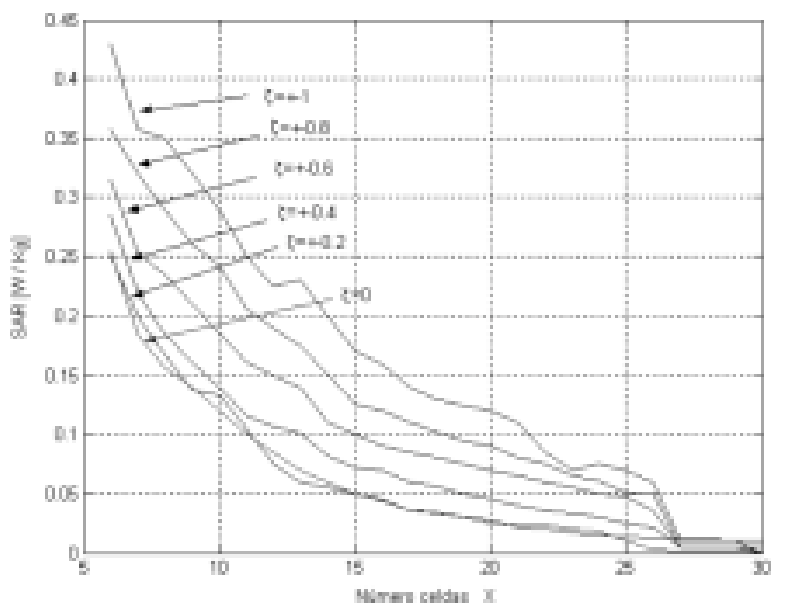

Fig. 6 SAR en función de $\zeta= \pm 1$.

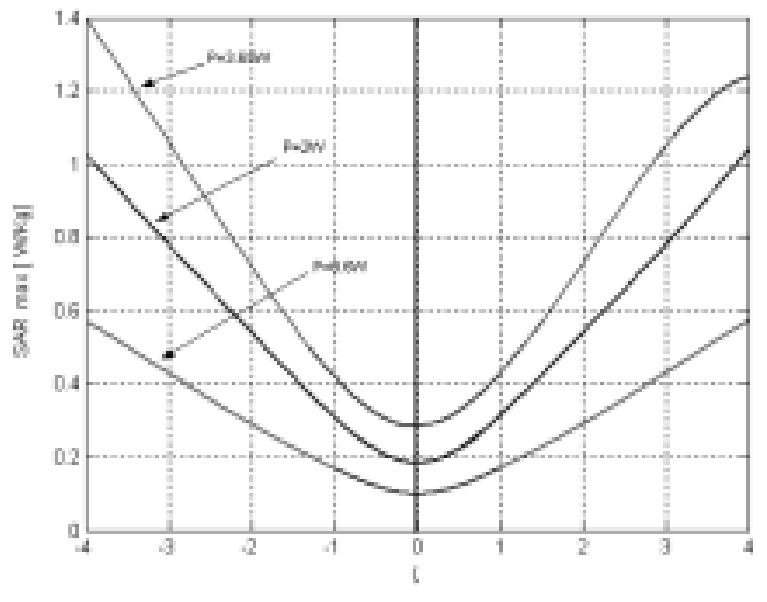

Fig. 7 Variación del SAR máximo en función de $\zeta$.

La segunda parte de las simulaciones son las correspondientes a las capas $11,12,13$ y 14 , cuyas imágenes digitalizadas se muestran en la Fig. 8. Para las capas 11, 12 y 14 la variación del SAR es similar a los gráficos de la capa 35 (Figs. 3 a 7), es decir, el SAR disminuye aproximadamente en forma exponencial hacia el interior de la cabeza. En cambio, para la capa 13 se observa, además de la respuesta estándar, una respuesta de carácter impulsiva, lo que corresponde a los denominados puntos calientes (hot point), puntos de alta absorción, lo que se puede observar en las Figs. 9 a 11.

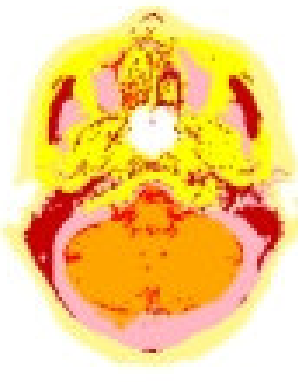

Capa 11

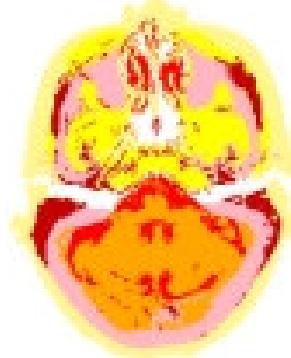

Capa 13

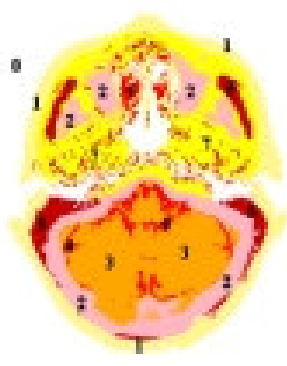

Capa 12

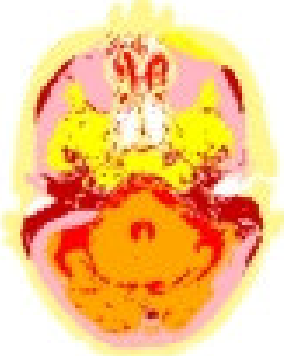

Capa 14
Fig. 8 Imagen digitalizada capas 11-14. Matriz 100x100. 


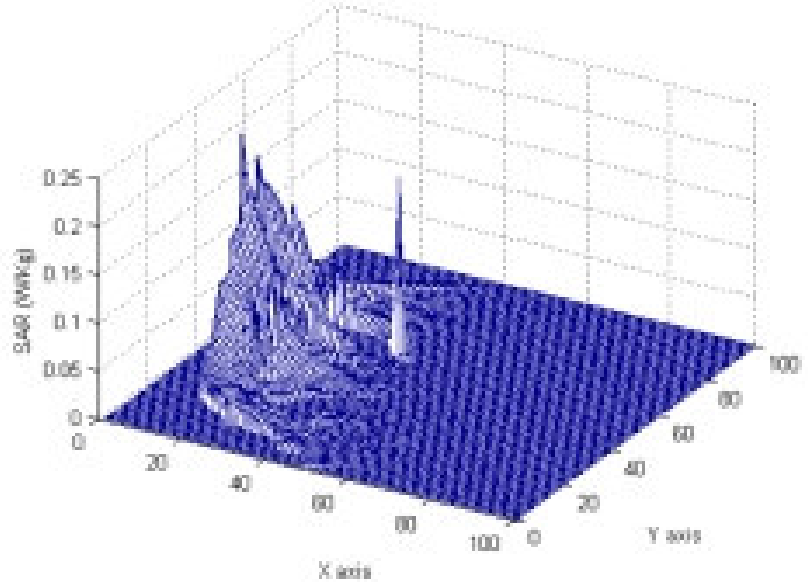

Fig. 9 Distribución del SAR en la capa 13 para $\zeta=1$.
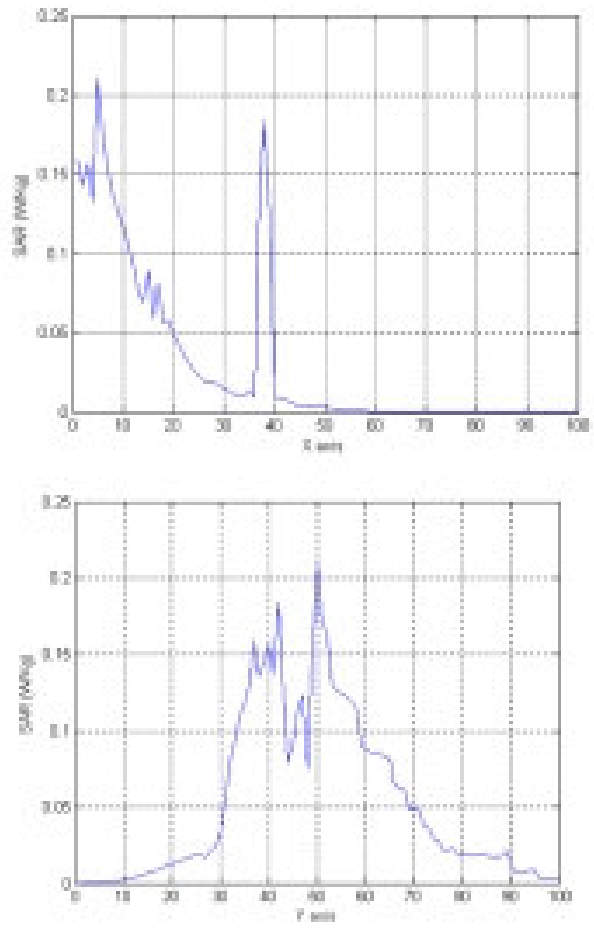

Fig. 10 Perfiles del SAR en la capa 13 para $\zeta=1$.

Este punto caliente se genera debido a que la radiación alcanza una región del cerebro en forma casi directa a través de los canales auditivos, lo que se observa analizando la estructura interna de la capa 13 en la Fig. 8; en cambio, los otros cortes $(11,12$ y 14) muestran la región cerebral protegida por más medios (músculos y huesos) y, por tanto, la absorción es menor. El valor máximo de este punto caliente se ubica en las coordenadas $(40,51)$ para un factor quiral unitario (ver Fig. 9) y el efecto de este factor sobre la magnitud del punto caliente se observa en la Fig. 11, en la cual hemos graficado el valor máximo del SAR para las 4 capas.

Respecto de la respuesta en la capa 13 se concluye que: 1) el comportamiento del SAR máximo es muy diferente al de las otras capas, debido a la existencia del punto caliente en ella, y 2) el efecto de la quiralidad sobre el SAR máximo es mucho más pronunciado que en los otros casos. Para efectos comparativos se ha graficado el valor del SAR máximo, para las 4 capas, a la entrada de la cabeza, o sea, para las coordenadas $\left(0, \mathrm{Y}_{\max }\right)$; aquí se observa que las todas las capas, de la 11 a la 14 , tienen un comportamiento similar. Todas las simulaciones del SAR se obtuvieron para una potencia de $3,65 \mathrm{~W}$ y una impedancia de antena de $180 \mathrm{ohms}$.

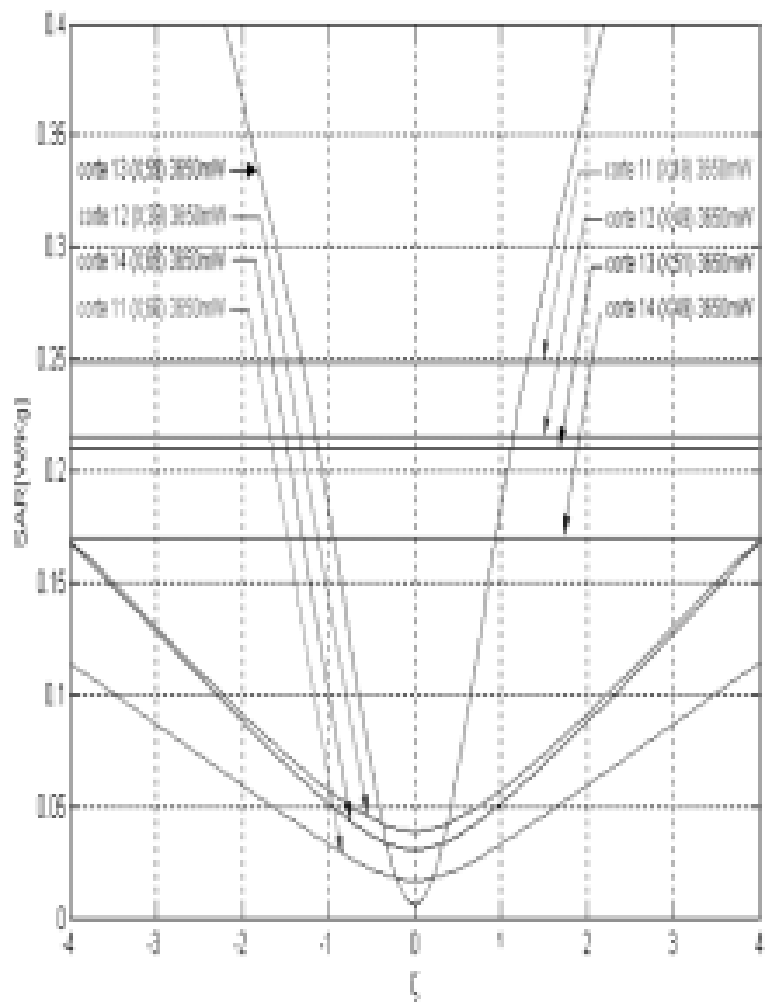

Fig. 11 Variación del SAR máximo en función de $\zeta$.

\section{CONCLUSIONES}

Se ha presentado un modelo bioplasmático quiral de la cabeza humana, que permite determinar y evaluar la absorción inducida por la radiación de teléfonos celulares. Primeramente se determinaron los campos electromagnéticos radiados mediante la técnica FDTD y luego el coeficiente de absorción específica SAR. Se 
ha demostrado que el uso de imágenes de resonancia magnética (MRI) en conjunto con el modelo de celdas (FDTD) y teniendo en consideración la quiralidad de las neuronas cerebrales, permiten determinar de una manera más precisa los campos cercanos inducidos en la cabeza por la radiación de microondas durante el uso de teléfonos celulares.

Primeramente, para la capa 35, se verificó a través del modelo clásico (aquiral) que la potencia absorbida por la cabeza disminuye cuando aumenta la distancia entre la fuente emisora (celular) y la cabeza y que el SAR aumenta si aumenta la resistencia de la antena. Usando el modelo propuesto, bioplasmático-quiral, se determinó por simulación la distribución del SAR cuando se considera la quiralidad del cerebro. Los resultados muestran que la potencia, absorbida por la cabeza, aumenta con el factor quiral. Respecto del caso aquiral $(\zeta=0)$, se tiene que para $\zeta= \pm 1$ el aumento es de 68 , para $\zeta= \pm 3$ el aumento es de casi 3 veces y para $\zeta= \pm 4$ es de 5 veces.

Otra conclusión muy importante es el descubrimiento de un punto caliente en una región del cerebro, en la capa 13 . Este resultado fue verificado haciendo simulaciones sistemáticas tanto en la capa 13 como en las capas vecinas. Se demostró también que la magnitud de este punto caliente aumenta considerablemente para elevados valores del factor quiral $(\zeta>1)$.

Estos resultados preliminares son significativos, porque permiten demostrar que, teniendo en cuenta la quiralidad del cerebro, aumenta la absorción, por parte de la cabeza humana, de la radiación emitida durante el uso de los teléfonos celulares. Será necesario corroborar la importancia de estos primeros resultados mediante modelos más avanzados. Por ello la continuación de nuestras investigaciones se orientará hacia el uso de un modelo bioplasmático-quiral tridimensional, considerando todos los cortes transversales, es decir, el total de las 50 capas. De éstas tienen particular importancia estudiar las capas en las cuales se ubican los ojos (en torno de la capa 25), ya que éstos órganos, al tener escasa irrigación sanguínea, son más vulnerables a los efectos térmicos (y no-térmicos) producidos por la absorción de microondas. Por otra parte, se incluirá, en el modelo propuesto, el efecto del campo magnético producido por la corriente de la batería del teléfono celular.

\section{AGRADECIMIENTOS}

Este trabajo ha sido financiado, parcialmente, por el proyecto Fondecyt-Chile $\mathrm{N}^{\mathrm{o}} 1040744$ y por los proyectos UTA-Chile Nos 8721-03 y 8722-03.

\section{REFERENCIAS}

[1] M.D. Taurisano and A. Vander Vorst. "Experimental Thermographic Analysis of Thermal Effects Induced in a Human Head Exposed to $900 \mathrm{MHz}$ Fields of Mobile Phones". IEEE Trans. on MTT. Vol. 48, No 11. November 2000.

[2] Research Coordinate Committee, Office of Global and Integrated Environmental Health. "International EMF Project: Health effects of static and time varying electric and magnetic fields". World Health Organization. Geneva, Switzerland. December 1997.

[3] F. Apollonio, M. Liberti, G. d'Inzeo and L. Tarriconi. "Integrated models for the Analysis of Biological Effects of EM Fields Used for Mobile Communications". IEEE Trans. on MTT. Vol. 48, $\mathrm{N}^{\mathrm{o}}$ 11, pp. 2082-2093. November 2000.

[4] H. Torres y M. Zamorano. "SAR simulation for chiral waves in head model”. Rev. Fac. Ing.-Univ. Tarapacá. Vol. 11, pp. 3-11. 2003.

[5] H. Torres Silva. "Waves in a Chiral-Plasma Media". The Japanese Journal of Physics. Vol. 67, pp. 850857. 1998.

[6] H. Torres Silva. "Propagación de Ondas Pulsadas en un Chiroplasma Magnetizado". Rev. Mexicana de Física. Vol. 44, No 3. December 1998.

[7] M. Zamorano y H. Torres Silva. "Ecuación de Schrödinger para una Fibra Óptica Quiral”. Revista Mexicana de Física. Vol. 46, $\mathrm{N}^{\mathrm{o}}$ 1, pp. 62-66. Febrero 2000.

[8] H. Torres Silva and M. Zamorano. "Chiral Effect on Optical Soliton". The Journal Mathematics and Computers in Simulation. Vol. 62, pp. 149-161. 2003.

[9] M. Zamorano y H. Torres Silva. "Efecto de la quiralidad sobre solitones polarizados en un medio anisotrópico”. Rev. Mex. de Física. Vol. 49, № 1. Febrero 2003.

[10] Y. Rahmat-Samii. "Real Time Simulation of the Interaction of Electromagnetic Waves with a Human Head". Dirección Web: http://www.ee.olemiss.edu/atef/headpresentation/ sld001.htm, May. 2003. 
[11] J. Paul. "Modeling of General Electromagnetic Material Properties in TLM". Doctoral Thesis. University of Nottinghan. UK. Chapter 6. 1998.

[12] A. Taflove and S. C. Hagness. "Computational Electrodynamics: The Finite-Difference Time Domain Method". Artech House. Second Edition. Chapter 6, pp. 248-251. 2000.

[13] H.Y. Chen. "Current and SAR Induced in a Human Head Model by the Electromagnetic Fields Irradied from a Cellular Phone". IEEE Trans. MTT. Vol. 42, No 12, pp. 2249-2254. December 1994.
[14] G. Lazzi. "Use of PML Absorbing Layers for the Truncation of the Head Model in Cellular Telephone Simulations". IEEE Trans. on MTT. Vol. 48, № 11, pp. 2033-2039. November 2000.

[15] A. Schiavoni, P. Bertotto, G. Richiardi and P. Bielli. "SAR Generated by Cellular Phones-Phone Modelling, Head Modelling and Measurements". IEEE Trans. on MTT. Vol. 48, N 11, pp. 2064-2071. November 2000. 